\title{
ANÁLISIS DE RESPONSABILIDAD POR DAÑO ESPECIAL A LA LUZ DE LA PONDERACIÓN Y LA TEORÍA DE LA PREVENCIÓN DEL DAÑO
}

\author{
Carlos Fernando Amaya Rodríguez \\ Recibido: Septiembre 29 de 2015 \\ Aprobado: Noviembre 30 de 2015
}

\section{RESUMEN}

En el campo del Derecho Administrativo la responsabilidad patrimonial del Estado es uno de los temas con mayor tensión jurídica. De igual forma no debe perderse de vista que la fuente de este sistema de responsabilidad estatal proviene de las reglas de imputación y atribución del derecho civil. Así pues al resolver si el Estado tiene que responder con ocasión de la ocurrencia de un daño antijurídico, esto será el resultado de la presencia de una serie de factores que formalmente le imputen el deber de reparar el daño causado, para ello se ha fijado un sistema de responsabilidad objetiva y subjetiva, cada uno de ellos con especialidades en lo que al factor de atribuación refiere, por ejemplo, en la responsabilidad obejtiva se tiene el daño especial, entendido como la ocurrencia de responsabilidad de reparar un daño causado a un particular en el marco de una actuación legítima del Estado. Resolver esta tensión entre el ejercicio legítimo de las funciones del Estado y los derechos particulares no solo será el resultado de exponer los elementos de la responsabilidad en la ecuación (daño, nexo y actuación), sino de la aplicación de métodos de resolución de conflictos entre derechos y

\footnotetext{
* Abogado de UNICOC, Especialista en Contratación Estatal de la Universidad de la Sabana y estudiante de los cursos de Doctorado de la Universidad de Buenos Aires. Actualmente se desempeña como Secretario Académico (E) del Colegio Jurídico y de Ciencias Sociales de La Institución Universitaria Colegios de Colombia UNICOC así como titular de las cátedras de Derecho Administrativo General II y Contratación Estatal de la misma casa de estudios. En la investigación es miembro del Grupo de Investigaciones Socio Jurídica de UNICOC GISU en la línea de Derecho, Estado y Sociedad, así mismo es líder del Semillero de Investigación del Colegio Jurídico y de Ciencias Sociales denominando "Retos, Innovaciones y Perspectivas del Derecho Administrativo” UNICOC.correo electrónico: cfamaya@ unicoc.edu.co
} 
principios, tales como la ponderación o las nuevas tendencias que se importan de la teoría de la prevención del daño.

Palabras claves: Responsabilidad, Función Administrativa, Daño Especial, Ponderación, Prevención del riesgo

\title{
AN ANALYSIS OF THE RESPONSIBILITY OF THE STATE IN THE CASES OF ILLEGAL DAMAGES FROM THE PERSPECTIVE OF THE BALANCING AND WEIGH THEORY AND THE DAMAGE PREVENTION THEORY
}

\begin{abstract}
Within Administrative Law, the patrimonial responsibility of the State is one of the topics that entitles most legal tension. It cannot be ignored that the rules of imputation and attribution of civil law constitute the main source of the system of the responsibility of the State. Therefore, the responsibility of the Sate in case of an antilegal damage will depend on the existence of several factors that make it responsible for the repair of damage. There is a system of objective and subjective responsibility, each one with special features as far as the attribution is concerned. For example, the objective responsibility considers the special damage as the responsibility to repair a damage caused to an individual due to a legitimate act of the State. In order to solve the tension between the legitimate exercise of the functions of the State and the rights of individuals it will be necessary to consider the elements of the responsibility within the equation (damage, connection and act), and to apply methods for resolving conflicts between rights and principles, such as weighing or the new tendencies imported by the theory of the prevention of damage.
\end{abstract}

Key words: Responsibility, Administrative function, Special damage, Weighing, Prevention of risk

\section{ANÁLISE DA RESPONSABILIDADE POR DANO ESPECIAL À LUZ DA PONDERAÇÃO E DA TEORIA DA PREVENÇÃO DO DANO}

\section{RESUMO}

No campo do direito administrativo da responsabilidade patrimonial do Estado é uma das questões com maior tensão jurídica. Não deve ser 
esquecido que a origem do sistema de responsabilização do Estado vem das regras de imputação e atribuição do direito civil. Assim, para decidir se o estado tem de responder à ocorrência de um dano ilegal, este vai ser o resultado da presença de um número de fatores que imputados formalmente direito para reparar os danos causados, por isso tem sido definido sistema responsabilidade objetiva e subjetiva, cada um com especialidades em que o coeficiente de atribuição está em causa, por exemplo, na responsabilidade objectiva se tem o dano especial, entendida como a ocorrência de responsabilidade de reparar os danos causados a uma pessoa em âmbito de uma ação estatal legítima. Resolver essa tensão entre o exercício legítimo das funções dos direitos estatais e privadas não só será o resultado de expor os elementos da responsabilidade na equação (dano, nexo e atuação), mas a aplicação de métodos de resolução de litígios entre direitos e princípios, tais como a ponderação ou as novas tendências importadas a partir da teoria da prevenção do dano.

Palavras-chave: Responsabilidade, Serviço administrativo, Dano/lesão especial, Ponderação, Prevenção de riscos

\section{INTRODUCCIÓN}

La Constitución Política colombiana de 1991 fundamentó sin lugar a dudas un sin número de aspectos relativos al Estado y al ejercicio de sus funciones, en especial el deber de reparar del Estado cuando con su actuar o no, se generan daños o lesiones a los bienes jurídicos de los administrados. En el marco de lo anterior se ha configurado un régimen especial de responsabilidad para las actuaciones del Estado, que incluso en el ejercicio de funciones legítimas de este último son causales de daños o afectaciones. Este sistema se consolida en el desarrollo de unos títulos de atribución o imputación de responsabilidad del daño y por consecuente el deber de reparar al Estado, los cuales se decantan sobre una ecuación de responsabilidad que contiene las variables de: daño, nexo de causalidad, y actuación (omisión) administrativa, que de cumplirse con los preceptos de la norma, configuran la declaratoria de responsabilidad patrimonial del Estado Colombiano y la obligación de reparar.

Ahora bien, se presentan casos en los que en principio, el desarrollo de la actuación administrativa como fuente de la responsabilidad es ejecutada 
en el marco de la legalidad, por lo cual no cabría la declaratoria en responsabilidad ya ampliamente expuesta. En armonia con lo anterior se constituye el factor o título de imputación del daño especial, el cual en términos generales permite la imputación de responsabilidad al Estado, cuando con su actuación se desequilbran las cargas públicas que todos los asociados estan en el deber de soportar en el ejercicio de una actuación légimita.

Es precisamente en dicho desequilibrio en donde entran en tensión o conflicto los principios y derechos tanto del Estado como de sus asociados. Así las cosas y como ya se expuso, la declaratoria de responsabilidad patrimonial es el resultado de advertir el desquilibrio entre las cargas públicas. A efectos de lo anterior, es entonces necesario recurrir a los métodos de interpetación de la norma y, a la construcción de juicios con el fin de advertir la presencia o no del desequilibrio de las cargas públicas.

En el presente trabajo se pretenderá desarrollar el método de ponderación de derechos como ejercicio para la solución del problema jurídico presente en la sentencia que será objeto de estudio. Dicha ponderación se realizará en dos ejercicios; uno de ellos encaminando a ser resuleto de tal forma que compagine con la sentencia objeto del estudio, y otro en donde el resultado sea completamente diferente. Es interesante reconocer que dependiendo de la argumentación que se otorgue a cada una de las posiciones, el ejercicio de ponderación correspondiente fijará el resultado que pretenderá el operador jurídico según sea el caso.

A efectos de tener otra visión de cómo resolver el conflicto de derechos, se relizará en este trabajo una revisión del caso de la Sentencia del Consejo de Estado en donde existe una revisión respecto de una presunta responsabilidad por daño especial, bajo la óptica de la teoría de la prevención del daño como explicación de la declaratoria de responsabilidad del Estado, como visión contrapuesta a la ponderación de derechos y principios.

Finalmente se realizará una crítica al fallo del Consejo de Estado objeto de análisis respecto a la declaratoria de inhibición para pronunciarse de fondo sobre el petitun, después de haber realizado todo un ejercicio por parte del Consejo de revisión de la jurisprudencia, doctrina y reglas aplicables al caso en comento. 
El Estado Colombiano a partir del año 1991 con la expedición del texto constitucional, reconoce el deber de la Adminsitración de reparar patrimonialmente los daños antijurídicos causados con ocasión del ejercicio u omisión de sus funciones. Bajo dicho postulado se construye la responsabilidad del Estado por daño especial como un marco lógico para la reparación del daño causado a un particular en el marco de una actividad legítima, motivo por el cual surge le necesidad de advertir si ¿el método de ponderación de principios es el adecuado para resolver el deber o no de reparar los daños antijurídicos causados a un particular en el marco de una actuación legítima de éste?

Se partirá de la hipótesis de trabajo de que la ponderación es un método de resolución de conflictos entre principios en donde se obtienen diferentes resultados según la carga y enfoque argumental que el operdador jurídico imprima en la solución del caso, lo cual produce una sentencia con diversos resultados, generando incertidumbre sobre la legitimidad del resultado de la ponderación expresada en el fallo y aun más, llegando a ser la puerta para la construcción de fallos inhibitorios del máximo Tribunal de lo Contencioso Adminsitrativo.

El presente trabajo se ha desarrollado principalmente bajo el método análitico deductivo, es decir, partiendo de los postulados generales del Derecho Adminsitrativo y la Responsabilidad del Estado, para llegar a conclusiones específicas en los casos puntuales del área de conocimiento del daño especial. De igual forma se tomará como referencia de caso de estudio una sentencia del Consejo de Estado relativa a la responsabilidad por daño especial a fin de identificar los factores de atribución o titulos de imputación de la responsabilidad, así como reconocer los derechos y principios en conflicto, para luego realizar un ejercicio de ponderación de principios para resolver el caso, buscando obtener un producto relativo al fallo y uno completamente diferente, como una metodología que advierte multiplicidad de opciones y resultados ofrecidos por la ponderación. De la misma manera se desarrollará un ejercicio aplicativo de la teoría de la prevención del riesgo, en algunos momentos se acudirá al método del derecho comparado, con el fin de realizar un símil entre las experiencias jurídicas colombianas y argentinas.

La selección de la sentencia objeto de analisis será tomando como criterio para la presencia de una revisión de responsabilidad cuyo factor de atribución sea la responsabilidad objetiva por daño especial. 


\section{GENERALIDADES DEL CASO OBJETO DE ESTUDIO EN LA SENTENCIA DEL CONSEJO DE ESTADO CONSEJO DE ESTADO, 8 DE MARZO DE 2.007, EXPEDIENTE NO. 16421, CONSEJERA PONENTE DRA. RUTH STELLA CORREA, BOGOTÁ.}

Con el fin de desarrollar el análisis propuesto en la metodología, se presenta el siguiente caso de responsabilidad extracontractual del Estado Colombiano, en donde por medio de la acción (hoy en día bajo la Ley 1437 de 2.011 denominado medio de control) de reparación directa, se pretende por parte del actor la reparación de los daños causados por la expedición de un acto administrativo, que a juicio de él es la fuente de causación de un verdadero daño antijurídico.

El accionante procede a demandar al municipio de la Virginia (Departamento de Risaralda Colombia) y a su alcalde. Toda vez que con la expedición del acuerdo No. 006 de 1.996 el Concejo de esta entidad territorial se aprobó la supresión de la Contraloría Municipal de la Virginia, de la cual el actor de la demanda era el titular del despacho para aquel entonces.

El centro de los argumentos del demandante se encuentran en los entendidos de que si bien el Alcalde y el Concejo cumplieron los mandatos y trámites legales para la supresión del citado órgano de control fiscal, esta situación generó un desequilibrio en las cargas públicas que deben soportar los administrados, así como también los mismos funcionarios de la administración (por la especial relación de sujeción que entre la Administración y sus funcionarios existe) toda vez que a juicio del accionante la Administración no tomó las medidas necesarias para mitigar los riesgos de causación de daños y perjuicios con ocasión de la expedición de su acto. El accionante durante todas las etapas procesales expuso que lo que se pretendía no era la anulación del acto administrativo sino por el contrario, la reparación de los daños especiales que surgen con ocasión de esa actividad legítima de la Administración.

El demandado por su parte desestimo la demanda y su Petitum, aludiendo que el Acto Administrativo mediante el cual se suprimió la Contraloría municipal de la Virginia se efectuó dentro de los mandatos legales y el régimen de municipios contenidos en la Ley 136 de 1.994, ya que el citado régimen amparaba legalmente la actuación la administración frente a la contraloría municipal, máxime cuando el municipio estaba incurso en las justificaciones necesarias para la 
supresión del órgano de control, es decir, para la desaparición de los requisitos para su creación, de igual forma justifico su decisión en la incapacidad económica del municipio para soportar la estructura organizacional y funcional de la Entidad. Lo anterior es debidamente consignado en la motivación o consideración del Acto Administrativo; por lo que a juicio de la parte demandada la presunta responsabilidad que se le imputa no está llamada a prosperar toda vez que la actuación se dio en el margen de una actividad legítima y legal por parte de la administración.

En resumen, la demanda y su Petitum no prosperó ante el tribunal administrativo de Risaralda, el cual mediante sentencia negó las pretensiones. El actor haciendo uso de los recursos judiciales ordinarios apeló la decisión del tribunal ante el Consejo de Estado, el cual después de una exposición de la línea jurisprudencial y reflexiones sobre las teorías de la responsabilidad extracontractual objetiva del Estado, procedió a confirmar la sentencia recurrida, pero no bajo los mismos entendidos de tribunal de primera instancia, sino bajo el marco de una sentencia "inhibitoria."

\subsection{Factores de atribución.}

El caso expuesto en el apartado anterior se encuentra dentro de la Responsabilidad Objetiva extracontractual del Estado, encontrando su fuente en la Constitución Política de Colombia de 1.991 que en su art. 90, doctrinal y jurisprudencialmente es conocido como la cláusula general de la Responsabilidad Patrimonial del Estado. La citada norma impone el deber de reparar y responder por los Daños antijurídicos causados a los particulares por la acción u omisión del Estado (y sus funcionarios), que de igual forma, guarda amplia relación con el art. 6 del citado texto constitucional en el cual se exponen los regímenes de responsabilidad de los particulares (administrados) los cuales solo responderán por la infracción de la constitución y la ley, y los funcionarios públicos que además de responder por lo anterior también lo harán por la omisión y extralimitación de sus funciones.

Dentro de los regímenes de responsabilidad extracontractual del Estado colombiano se ha evidenciado dos tipos de responsabilidad, una de tipo subjetiva y otra de tipo objetiva. Para cada clase existen diferentes títulos de imputación de responsabilidad que para efectos de la doctrina y la jurisprudencia extranjera, como la argentina, le reconoce también como factores de atribución. Para el caso en concreto estamos en presencia de 
una presunta responsabilidad del Estado de tipo objetiva con ocasión de los hechos presentados en el apartado anterior.

Tomando como base los planteamientos anteriores se hace necesario exponer que para la citada responsabilidad objetiva existen dos factores o títulos de atribución de responsabilidad, siendo estos a saber: Daño especial y Riesgo Excepcional. Dentro de esta forma de responsabilidad se debe tener presente que el precepto rector del art. 90 del texto constitucional colombiano de 1991 de la causación de un daño antijurídico se debe desprender o ser consecuencia de una actuación de la Administración en el ejercicio de un actividad legitima del Estado, es decir amparada en el marco de la Ley, a fin de encajar en la categoría del daño especial.

Para el caso objeto de estudio y del texto de la sentencia se aprecia como título de imputación o factor de atribución el Daño Especial, en donde la responsabilidad de reparar el daño es atribuible a la Administración. Lo anterior en el entendido de que mediante una actuación lícita se genera un desequilibrio de las cargas públicas a las que están sometidos los administrados y los mismos servidores del Estado.

El centro de este factor se haya en la vulneración del principio de igualdad de las cargas públicas, así las cosas "mientras un ciudadano soporte las mismas cargas que los demás, nada puede reclamarle al Estado pero si llega a soportar una carga especial surge el deber de indemnizar a cargo de este último." (Ruiz, W. 2.013, p. 18) Estas cargas públicas a las que refiere la cita son resultado del modelo de Estado Social de Derecho en el cual se construye el Estado Colombiano, donde el interés general es el fin y deber primordial de este último en lo que refiere a la búsqueda, prestación y protección del citado interés, pero respetando racionalmente la intensidad en la que se deben soportar dichas cargas.

Así pues, cuando la actuación genera un daño a todos los asociados en el mismo nivel e intensidad, con ocasión de la búsqueda del citado interés general o colectivo, no genera responsabilidad para la administración, sin embargo, cuando uno de los administrados o incluso los mismos servidores públicos sufre un desbalance en lo que refiere a soportar las cargas se genera entonces la fuente de un daño antijurídico, ocasionando para el afectado, el derecho de realizar la respectiva reclamación ante el juez de lo contencioso administrativo a fin de obtener si prosperan sus pretensiones, la indemnización o compensación de los daños morales y materiales a los que dependiendo el caso hubiere lugar. 
Estamos en presencia entonces de una responsabilidad sin falla de la administración, es decir sin la presencia del elemento subjetivo que aporta el comportamiento del funcionario público encargado de la gestión directa o indirecta de la función administrativa del Estado, en donde, como ya se ha expuesto, es en el ejercicio legitimo de la gestión administrativa, pero que en todo caso lesiona de manera desequilibrada los derechos jurídicos o situaciones consolidadas de los administrados y en casos especiales de los mismos servidores públicos.

Es entonces lo especial del daño la causa de la responsabilidad de indemnizar por parte del Estado. El Consejo de Estado de Colombia ha expuesto con claridad este punto, en donde afirmo que

Responde el Estado, a pesar de la legalidad total de su actuación, de manera excepcional y por equidad, cuando el obrar de tal modo, en beneficio de la comunidad, por razón de las circunstancias de hecho en que tal actividad se desarrolla, causa al administrado un daño especial, anormal, considerable, superior al que normalmente deben sufrir los ciudadanos en razón de la especial naturaleza de los poderes y actuaciones del Estado, rompiéndose así la igualdad de los mismos frente a las cargas públicas, o a la equidad que debe reinar ante los sacrificios que importa para los administrados la existencia del Estado. (Consejo de Estado, 13 de diciembre de 2.005, Expediente No. 24.671 C.P. Alier Hernández Enríquez, Bogotá.)

En el desarrollo de las consideraciones de la sala en el marco de la sentencia objeto del presente análisis, se expone una importante revisión de la evolución jurisprudencial sobre la Responsabilidad del Estado cuando la fuente de ésta se encuentra enmarcada en el acto administrativo que es conforme al ordenamiento jurídico, es decir, libre de vicios. Aborda en un primer momento, que frente a los daños que causare el mencionado acto no eran susceptibles de reparación y muchos menos factores para atribuir responsabilidad al Estado. Así pues:

Muchos actos administrativos ocasionan desde el punto de vista material evidentes perjuicios a personas de derecho público o privado, pero ellos no pueden considerarse dentro de la técnica jurídica como tales. Es ese el caso, por ejemplo, de la creación de los nuevos municipios, segregándolos de los existentes. Es indudable que el distrito mutilado 
experimenta daños notorios. Pero ellos no pueden tomarse en cuenta, porque el beneficio general de la colectividad, que resulta del acto, los hace inoperantes y no permite contemplar sino los aspectos favorables que trae consigo el ejercicio de la competencia que tienen las asambleas para crear los nuevos municipios (Consejo de Estado, 7 de abril de 1.938, C.P. Carlos Lozano y Lozano)

Se evidencia del extracto anterior que la ausencia de responsabilidad del Estado se justificaba en el principio de la prevalencia del interés general que debe buscar toda manifestación de la función administrativa de los Estados (sobre este principio ahondaremos más adelante).

Posterior a ello el Consejo de Estado reorganiza la posición jurisprudencial en el marco de la existencia de responsabilidad para los casos en los cuales se produjere un daño con ocasión de la expedición de un acto administrativo conforme a derecho y sus consecuentes efectos, bajo el argumento de la ruptura del equilibrio de las cargas públicas que se deben soportar. Dentro del fallo hito en donde hace el cambio de la posición del Consejo de Estado se pone de presente que el medio idóneo para reclamar la indemnización para estas situaciones no es la acción de nulidad y restablecimiento del Derecho sino la Reparación Directa, por ello

La Sala estima que el presunto daño que se puede causar por un acto administrativo legal no puede reclamarse a través de la acción de nulidad y restablecimiento del derecho, como la incoada en el presente caso, dado que el restablecimiento del derecho en ésta tiene, por el contrario, fundamento exclusivo en la inconstitucionalidad o ilegalidad del acto. Tal pretensión solamente podría deducirse ante esta jurisdicción mediante la acción de reparación directa en la modalidad de responsabilidad extracontractual del Estado por daño especial ${ }^{1}$.

1 CONSEJO DE ESTADO, SALA DE LO CONTENCIOSO ADMINISTRATIVO, SECCIÓN PRIMERA, Sentencia de 21 de marzo de 1996, Rad. 3575, Actor: Sociedad Las Mercedes Ltda. Sucesores y Cía. S. En C. S.,C. P. Ernesto Rafael Ariza Muñoz. Criterio reiterado en SECCIÓN PRIMERA, Sentencia de 28 de noviembre de 1996, Rad. 3967, Actor: Hacienda Villa Lucía Limitada Martha Patricia Ramírez Nieto, C. P. Libardo Rodríguez Rodríguez; SECCIÓN PRIMERA, Sentencia de 8 de mayo de 1997, Exp. 4291, Actor: Kokoriko Ltda., C. P. Juan Alberto Polo Figueroa; SECCION PRIMERA, Sentencia de 8 de mayo de 1997,Rad. 4208, Actor: Sociedad Operaciones Bursátiles S.A., Demandado: Concejo Municipal de Santiago de Cali, C. P. Juan Alberto Polo Figueroa; SECCIÓN PRIMERA, Sentencia de 22 de mayo de 1997, Exp. 4261, Actor: Antonio Obeso de Mendiola y Otra, C. P. Ernesto Rafael Ariza Muñoz y SECCION PRIMERA, Sentencia de 22 de mayo de 1997, Rad. 4207, Actor: Sociedad Las Mercedes Ltda. Hermanos y Cía. S. en C.S., Demandado: Concejo Municipal de Santiago de Cali, C.P. Libardo Rodríguez Rodríguez; SECCIÓN PRIMERA, Sentencia de 22 de mayo de 1.997, Rad. 4205, Actor: Yolanda Velasco de Navia y otros, Demandado: Concejo Municipal de Santiago de Cali, C.P. Ernesto Rafael Ariza Muñoz. 
Lo anterior es sana lógica pues lo que se pretende no es la anulación del acto, sino la reparación del daño causado de manera especial al demandante.

Prosigue con la presentación y justificación del daño especial, como elemento indispensable para la atribución de responsabilidad al Estado frente a situaciones tan especiales como las del caso objeto de estudio. El eje de la responsabilidad radica en el hecho de que la actuación genera un desequilibrio en las cargas que deben asumir los particulares, la jurisprudencia de esta Alta Corte de lo Contencioso Administrativo ha definido como daño especial

Aquel que se inflige al administrado en desarrollo de una actuación legítima del Estado ajustada en un todo a la legalidad pero que debe ser indemnizado por razones de equidad y de justicia distributiva, en la medida en que aquel se ha beneficiado a costa de un daño anormal, desmesurado o superior a aquel que debe soportar normalmente el administrado. (Consejo de Estado, 8 de marzo de 2.007, Expediente No. 16421, Consejera Ponente Dra. Ruth Stella Correa, Bogotá).

En conclusión, el factor de atribución correcto para el caso expuesto en la sentencia y objeto del presente análisis es el daño especial. Tomando como referente la exposición del concepto de este factor de atribución y los elementos de los que se compone, se encuentran presentes en el ya citado caso; pues el presunto daño que se le causó al actor de la demanda ha sido en el margen de una actuación legitima de la Administración que incluso encuentra su amparo en el marco de la Ley que regula el régimen de los municipios en Colombia, y como manifiesta el actor de la demanda, con ésta no se pretende obtener la anulación del acto administrativo ni cuestionar su legalidad sino el desequilibrio en las cargas públicas que se han causado con ocasión del legítimo ejercicio de la gestión de lo público por parte de la administración, y que ha generado un presunto daño antijurídico en la lesión de bienes jurídicos sin el deber de soportarlo, esto expuesto al suprimir la entidad de la Contraloría Municipal de la Virginia en el Departamento de Risaralda.

\subsection{Principios en conflicto.}

Para el desarrollo de este punto procederemos en primer lugar, a identificar los principios presentes en el marco de la sentencia objeto de 
análisis, posteriormente una explicación general de cada uno de ellos, y finalmente la exposición del conflicto de estos dentro del caso.

Con base al plan de trabajo propuesto se procederá a identificar los principios que se hacen presentes en el caso sometido a análisis:

a. Principio de la confianza legitima.

b. Principio de la prevalencia del interés general.

c. Seguridad jurídica.

Ahora bien, se expondrá de manera sucinta las definiciones categóricas que se decantan para cada principio identificado en el apartado anterior.

La confianza legítima como el primero de los principios en conflicto que se identifican en el caso objeto de estudio, radica en que el administrado tiene la seguridad absoluta de que las actuaciones de la administración son el resultado de un cuidadoso análisis y preparación de sus actuaciones, en donde se han identificado todos los riesgos del ejercicio de la función administrativa, buscando no causar desequilibrio en el peso de las cargas públicas que deben asumir los administrados.

El citado principio encuentra su origen en el artículo 83 superior (Constitución de Colombia de 1.991) en donde se garantiza la presunción de la buena fe en las actuaciones tanto de los particulares como de la Administración "las actuaciones de los particulares y de las autoridades públicas deberán ceñirse a los postulados de la buena fe, la cual se presumirá en todas las gestiones que aquellos adelanten ante éstas."

La Corte Constitucional Colombiana en su rica jurisprudencia ha analizado y expresado las implicaciones y alcances de la definición de este principio, en donde la buena fe se convierte en la base la confianza de los administrados de que la administración actuara de manera correcta y conforme a derecho por ello:

"La Corte Constitucional ha indicado que es deber de la administración actuar en sus relaciones jurídicas con los particulares de manera consecuente con sus conductas precedentes, de manera que los administrados no se vean sorprendidos con conductas que resulten contrarias a sus expectativas legítimamente fundadas." (Corte Constitucional Colombiana, Sentencia T 895, 2010) 
De igual forma esta Alta Corte expone que si bien la confianza legítima no es una especie de petrificación e inimputabilidad de las relaciones jurídicas entre el Estado para con sus servidores y administrados, empero si es de su deber velar por el respeto de los derechos adquiridos, y que los cambios a efectuar no sean de sorpresa para los administrados "de este principio no se puede derivar intangibilidad e inmutabilidad en las relaciones jurídicas que generan confianza para los administrados; respetando los derechos adquiridos y frente a situaciones susceptibles de modificación, el cambio de enfoques y entendidos no puede suceder de forma abrupta e intempestiva, debiendo la administración asumir medidas para que la variación que sea justa e indispensable, suceda de la forma menos traumática para los afectados." (Corte Constitucional Colombiana, T895, 2010)

Finalmente este principio gurda especial relación con el interés general, toda vez que se confía de manera legítima que toda actuación de la Administración está encaminada a la búsqueda, la protección del citado interés general, y como ya se mencionó previamente, debe mantener un balance en las cargas públicas (tema ya explicado en el apartado correspondiente al factor de atribución del daño especial).

La prevalencia del interés general resulta de la simple lógica que se desprende el concepto de ese interés. En primer lugar se debe mencionar que el interés general son aquellas situaciones que son comunes a toda la colectividad, por lo tanto es deber del Estado prestar atención a estas necesidades con el fin de brindar bienestar a la comunidad a su cargo.

Se construye entonces como un principio y faro rector del ejercicio y gestión de la función administrativa del Estado, tanto en la modalidad directa como indirecta, el cual encuentra su origen y fundamento en el texto constitucional en donde fue erigido como uno de los principios fundamentales del Estado Colombiano "Artículo No. 1. Colombia es un Estado social de derecho, organizado en forma de República unitaria, descentralizada, con autonomía de sus entidades territoriales, democrática, participativa y pluralista, fundada en el respeto de la dignidad humana, en el trabajo y la solidaridad de las personas que la integran y en la prevalencia del interés general" ; así pues se construyen los fines del Estado Colombiano que reposan en el artículo No. 2 del citado texto constitucional de 1.991 "Son fines esenciales del Estado: servir a la comunidad, promover la prosperidad general y garantizar la efectividad de los principios, derechos y deberes consagrados en la 
Constitución; facilitar la participación de todos en las decisiones que los afectan y en la vida económica, política, administrativa y cultural de la Nación; defender la independencia nacional, mantener la integridad territorial y asegurar la convivencia pacífica y la vigencia de un orden justo." Los cuales se pueden resumir en la esencia del Interés General.

Ahora bien, la seguridad jurídica es un principio que busca generar confianza y certeza sobre el derecho vigente y las obligaciones que se construyen en el marco de los ordenamientos jurídicos. Debe tenerse claro al igual que el anterior principio expuesto, esta certeza no genera la inmutabilidad del Derecho y las relaciones que este regula, pues de ser así se estaría desconociendo una cualidad del derecho, que no es más que la mutabilidad, adaptabilidad y evolución del derecho a las coyunturas sociales, históricas, económicas y culturales.

Sin embargo, esta seguridad jurídica busca dar claridad al panorama sobre el que debe estar preparado el particular frente a su relación con las diferentes entidades de la administración, sin recibir sorpresa sobre las reglas de juego y las disposiciones normativas aplicables a cada situación. La Corte Suprema de Costa Rica en su Sala Constitucional ha definido claramente este principio, en su palabras:

(...) la aaplicación del principio de seguridad jurídica, el Estado viene obligado a proveer un marco normativo para que el ciudadano sepa a qué atenerse en sus relaciones con la administración. Así, la seguridad jurídica en sentido estricto, no precisa tener un determinado contenido, sino que bastará con la existencia de un conjunto de disposiciones que fijen consecuencias jurídicas frente a ciertos hechos o actos. (Corte Suprema de Justicia de Costa Rica, Sala Constitucional, Sentencia No. 10375-11)

Es por ello que en el caso objeto de estudio el demandante se ha visto en una reforma al régimen de los municipios y más aún, en la aplicación de ese régimen en el municipio de La Virginia, afectando su estabilidad como titular del despacho de la contraloría de este Ente Territorial, el cual fue suprimido con ocasión del nuevo régimen jurídico.

Para concluir con este apartado, se estudiará a continuación el conflicto que se presenta entre cada uno de los principios expuestos previamente. 
En primer lugar el interés general se presenta como faro rector de toda actuación administrativa, así como de los respectivos efectos de las actuaciones, entre tanto la intención de la Administración del municipio de La Virginia fue proteger ese interés pues, a juicio de la entidad territorial, no era viable ni factible seguir con la carga económica que implicaba la contraloría municipal, lo que afectaba seriamente el tesoro público y por ende el Interés General que se manifiesta en la correcta y eficaz asignación y gastos de estos dineros. Lo anterior es un claro cumplimiento de la Administración a partir de una revisión a priori de la plataforma fáctica del caso, de su deber de buscar y preservar el interés general de la comunidad que tiene a su cargo.

El conflicto se presenta en el hecho de que la supresión de la Contraloría Municipal de la Virginia fue desarrollada a juicio del demandante, sin las respectivas precauciones e identificaciones de los riesgos y vulneración de derechos adquiridos al momento de la ejecutoria del Acuerdo del Concejo Municipal (Acto Administrativo) con el cual no solo se suprimió la contraloría, sino que adicional a ello se extinguieron todas las relaciones y situaciones jurídicas que dependían de la existencia de este órgano de control fiscal, expone como argumento el demandante que se incumplió con la seguridad jurídica y la confianza legitima, así pues "Señaló que esa responsabilidad es aún mayor, dado que el funcionario demandado, esto es, el alcalde de La Virginia, no adoptó las previsiones necesarias para indemnizar los daños causados a empleados de período fijo o de carrera administrativa, con la supresión de la dependencia (fls. 74 a 102 c. ppal.)" (Consejo de Estado, Expediente No. 16421, 2007)

Pareciere entoences que con la actuación amparada en la legalidad de la Ley que define el regímen de municipios en Colombia, y la protección al interés general (este ultimo como faro rector de la gestión administrativa del Estado), se han vulnerado los derechos de los funcionarios adscritos a la entidad suprimida sin la mayor previsión sobre su futuro inmediato ni las indeminzaciones respectivas; pues como se explicó previmanete es un deber de la Administración planificar el ejercicio de sus actividades a fin de evitar traumatimos a los particulares propendiendo por el respeto por los derechos adquiridos o identificando formulas de indeminzación cuando su afectación fueren imposibles de evitar.

\subsection{Ponderación.}

Es claro, que para relizar un ejercicio de ponderación es necesario que dos más principios se encuentren enfrentados o en colisión, y segundo, 
es necesario encontrar un balance entre los principios enfrentados a fin de obtener una resolución de la crisis, justificando las ventjas de la relización de unos de los principios enfrentados como compensación sobre los demás principios derrotados.

Es necesario dejar claro que el Consejo de Estado expresó que su sentencia es resultado de la revisión juiciosa de los argumentos del demandante. Pues si bien estan dados los presupuestos de la responsabilidad extracontractual del Estado al tenor de artículado constitucional de 1.991 y el título o factor de atribución es correcto, éste cuestiona de manera velada la legalidad del acto administrativo, por lo que no se está haciendo uso de la acción procesal adecuada para demandar la reparación de los daños; sin embargo, al no estarse atacando el acto adminsitrativo, se deduce que ha prosperado la prevalencia del principio de prevalencia del Interés General, el cual en todo caso ha sido la plataforma jurídica de la actuación administrativa causante del presente litigio.

Con forme a las consignas propuestas para el presente trabajo, se procederá a relizar dos ejercicios de ponderación, uno que ampare el resultado de la sentencia objeto de estudio y uno en donde el reusltado sea opuesto, aplicando las reglas de ponderación que consisten en analizar la idoniedad, necesidad y proporcionalidad de los principios enfrentados a fin de identificar uno que deba mediar a fin de conjurar el conflicto.

En el primer ejercicio, se ponderará de tal manera que el resultado sea acorde al resultado del fallo objeto de este analisis, es decir negando el petitum de la demanda y por ende la no reparación de los presuntos daños causados.

a. La idoneidad: El principio idóneo para ser defendido en este caso resulta ser la prevalencia del Interés General. Lo anterior en suma a todo lo expuesto ya que ésta prevalencia es el resultado obtenido al considerar al interés general como la principal fuente para le buena gestión de la función administrativa del Estado.

Por ello es que la actuación adminsitrativa se encuentra ajustada a derecho y no hay lugar a un desequilibrio de las cargas públicas que deben soportar los administrados, ya que el acto administrativo mediante el cual se suprime la Contraloria Municipal de la Virginia es completamente legítimo, y adicional a ello se invoca de manera 
adecuada la protección del tesoro público, pues era imposible para el municipio seguir soportando la carga prestacional y presupuestal.

De igual forma, es el medio idóneo para la protección de esta prevalencia, así como la realización del mandato que encierra el principio que resulta protegido. Pues sería incoherente con este mandato de orden constitucional, que se protegieren los interes particulares que sobre la supresión o no de este órgano de control fiscal pudise llegar a existir, máxime cuando lo que se busca con la cuestionada supresión no es más que porteger la eficacia en la asignación, distribución y ejecución del gasto de los dineros públicos a fin de poder se mas eficacies en la sastifación de las necesidades colectivas, tema que como ya se ha expuesto no es otro que el principal motor de desarrollo de las gestiones de los Estados Modernos.

En conclusión, por lo expuesto previamente y por la portección que se enmarca en un gasto público eficiente y eficaz a fin de atender las necesidades sociales, es que la supresión de la Contraloría Municipal de la Virginia resulta ser el medio idóneo para asegurar el principio de prevalencia del interés general sobre los demás principios.

b. La necesidad: Cuando se aborda este punto del juicio de ponderación, lo que se busca es, mediante una argumentación coherente demostrar por qué es necesaria la utilización del medio indicado para la protección del pincipio idóneo ya expuesto en la adecuación, sin que existiere otro medio para obtener el fin de optimizar el derecho que se encierra en torno al principio.

Así pues es claro que la supresión de la contraloría municipal de la Virginia bajo los entendidos de la motivación del acuerdo del Concejo Municipal del citado ente territorial, es más que necesario toda vez que está evidenciada la desapración de los presupuestos que justificaban la existencia del ente de control, así como la inviabilidad financiera del municipio para soportar la carga prestacional y de funcionamiento del ya ampliamente mencionado organo de control.

Del plenario de la sentencia se puede evidenciar que, la decisión y los efectos del acto son el medio para la salva guarda del interés general, pues como afirmó el demandado en su defensa se "Anotó que el municipio y el alcalde demandados se sujetaron a los mandatos legales, de manera que no se puede predicar responsabilidad alguna, dado que actuaron atendiendo la incapacidad económica del municipio para 
contar con su propio órgano de control fiscal, correspondiéndole ejercer dicho control a la Contraloría del Departamento (fl. 126 a 141 c. ppal.)." (Consejo de Estado, Expediente No. 16421, 2007); adicional a ello se puede conjurar en defensa del demandando que nadie estará obligado a lo imposible, así pues y demostrado que se estaría afectando el erario público y por ende el interés general. De igual forma el control fiscal del municipio no desaparecería sino que ahora estaría a cargo de la Contraloría Departamental.

En conclusión, y bajo los presupuestos fácticos que se tienen en torno al caso, es más que necesario la supresión de la Contraloría Municipal de la Virginia a fin de no generar afectación al gesto público, la atención a las necesidades sociales y por ende al Interés General.

c. La propocionalidad: Ahora bien reuslta indispensable identificar que realizar el principio de la prevalencia del interés general, resulta altamente beneficioso a la comunidad, pues con la liberación de las cargas económicas para el municipio que implica el funcionamiento de la contraloría, se puede reasignar el dinero en cuestión a proyectos que generen mayor impacto social (sasifacción de necesidades colectivas), o en otra eventualidad no se afectaría con la escaces de dineros por parte del municipio para el funcionamiento del órgano de control, así como otras dependencias y poryectos que tenga a su cargo el ente municipal de La Virginia. Por lo que es más que evidente que ante tales venturas resultaría ilógico que se mantuviere la estabilidad jurídica que se encierra en el marco de los principios de la confianza legítmia en las actuaciones de la administración y el principio seguridad jurídica. Así catalogado como fuerte el principio de la prevalencia del Interés General.

Como segundo ejercicio se procederá a relizar la ponderación de tal manera que el resultado sea contrario al anterior, es decir dando como reusltado el acceso al petitum demandado y la consecuente reparación.

a. La idoneidad: Es claro que deberá prevalecer los principios de la confianza legítima de la adminsitración, así como el de seguridad jurídica, pues es un deber de la Adminsitración, que en el ejercicio de sus actuaciones, el desarrollo de sus potestades y demás inherentes deben simpre ser desarrolladas con el máximo cuidado y respeto por los derechos adquiridos, situaciones jurídicas consolidadas, relaciones jurídicas que tienen los amidnistrados y los mismos servidores de la Adminsitración frente a ésta. 
Como se expreso en la descripción de los principios, la adminsitración debe a toda costa, mitigar los riesgos cuando por medio de sus actuaciones en ejercicio de su autoridad de mando llegare a modificar las situaciones y derechos adquiridos de sus adminsitrados, y así lo ha dejado en claro el máximo órgano de lo contecioso amdinistrativo en Colombia, al exponer que es un deber del Estado planear sus actuaciones (actos, operaciones, contratos) y mitigar los riesgos que sean previsibles, dando como reusltado el deber de indeminzar los daños y riesgos que en primer lugar no fueren posibles de evitar y en segundo lugar reparar sin protesto alguno los riesgos identificados con ocasión del cumplimiento de su deber de planeación.

Ahora bien, el medio idóneo para cumplir con los anteriores postulados es un verdadero ejercicio de planeación y preparación de la voluntad de la Administración que se verá plasmada en el correspondiente acto, así pues es deber del municipio proceder a reparar el desequilibrio que generare su actuación en las cargas públicas, o en su defecto:

b. La necesidad: Es claro a partir de lo expuesto en la idoneidad, para el presente ejercicio, que la protección de la seguridad jurídica y la confianza legítima en las actuaciones de la administración a través del ejercicio riguroso de planificación de sus actuaciones de tal manera que le permita mantener dichos principios vigentes y en la eventualidad de que se vieran afectados, diseñar un plan o estrategia que mitigue al mínimo exponente el impacto, pues no es viable como ya expusimos, un desequilibrio injustificado de las cargas públicas que se deben soportar por parte de los administrados o los servidores de la administración, sin la respectiva reparación por los daños causados.

c. La proporcionalidad: En este punto resulta pertinente exponer las bondandes que representa la prevalencia de los principios ya expuestos, su protección se hace necesaria e idónea a fin de matener el equilibrio de las cargas públicas por parte de la administración que han de soportar de manera justa sus administrados y servidores.

Implica entonces que el respeto por los derechos adquiridos y las situaciones jurídicas consolidadas por parte de la Administración en el desarrollo de sus potestades de mando y gestión, es mucho menos dañino que recibir demandas en donde se solicite la reparación de los daños causados y por ende, ya no se sacrificarían los dos principios seleccionados sino que adicionalmente el interés general se verá atacado con la disminución del patrimmonio público. 
La afectación de los principios escogidos resulta intensa, si no se cumpliere con las disposiciones vigentes. Mientras que con una intervención razonada éstos se verían protegidos y el detrimento del principio opuesto en este caso, será irrelvante pues como ya se dijo previamente, reuslta beneficiado en algunos apartes.

En ambos casos del ejercicio de ponderación se debe advertir que con el resultado de cada uno, se fijan reglas para la apliacación de uno de los principios respecto de los otros, bajo la argumentación ofrecida para indicar que principio es indóneo proteger. (Clerico, L. 2.010). Entonces, en el primer ejercicio se establece que es el principal deber del Estado asegurar el interés general de su comunidad, mandato que no puede ser limitado por el principio de seguridad jurídica y confiaza legítima que predican los particulares, incluso por aquellos que ostenten la calidad de servidor público, máxime cuando con la posibilidad de que prevalezcan los intereses anteriores se afecte el patrimonio público y por consecuente la efectividad del Estado para salvaguradar el interés general.

Ahora bien, en el segundo ejercicio y bajo la idea de que el método de ponderación genera reglas para la prevelancia idónea de uno de los principios, se da la posición de respeto por los intereses del servidor público afectado con el acto administrativo objeto del la revisón por parte del Alto Tribunal Contecioso Administrativo, debe prosperar en razón a que su inboservacia afectaría aún más el interés general que a juicio de los demandados la actuación pretende salvaguardar; reglas que para cada interpretación y argumentación son completamente validas y aplicables, pero que en todo caso dejan en entre dicho las verdaderas motivaciones para la aplicación idónea de uno y la exclusión de otro principio en conflicto.

\section{OTRA SOLUCIÓN, LA PREVENCIÓN DEL RIESGO COMO TEORÍA PARA LA DETERMINACIÓN DE RESPONSABILIDAD.}

Conforme a la ruta de trabajo propuestas en las consignas, se procederá a resolver el caso mediante otra posibilidad diferente a las reglas de ponderación. Reglas que como se puede apreciar en el ejercicio anterior, pueden permitir dos o más posiciones sobre un determinando caso según el enfoque que se le de a la balanza.

En el caso es claro que estamos ante un error de la administración pública, error que fue cometido en el ejericicio de la legimita acitivdad 
amdinistrativa, que empero fue generadora de un daño antijurídico especial (recordando la teoria que encierra el factor de atribución del daño especial), por lo que la solución que se propone como resultado de las situaciones fácticas la teoría de la prevensión del daño, en donde como correctamente expoene el Profesor Doctor Leandro Vergara, la obligación de reparar los daños es el resultado de cuando aun teniendo el deber de prevenir su ocurrencia la parte obligada no lo hace, o al menos no toma las medidas pertientes para mitigar el impacto del daño. (Vergara, L. 2.013) Veamos en este caso como se aplicarían los presupuestos de esta teoría para resolver el problema jurídico.

En primer lugar es necesario identificar los riesgos que representa el acto administrativo por el cual se suprime el órgano de control fiscal del municipio de la Virginia. Se deducen así dos riesgos evidentes; el primero de ellos la ruptura de las relaciones jurídicas y derechos adquiridos que versan sobre los funcionarios de la citada dependencia algunos con vinculación laboral amdinistrativa a témrino indefinido (funcionarios de carrera) y otros a término definido como lo es el caso del contralor municipal quien tiene definido su periodo de vinculación al mando del despacho. Ahora bien, si está presente la posibilidad de efectuar el ius variandi sobre estas situaciones, es deber de la Administración el mitigar el impacto de sus desiciones por medio de la previsión de una reacomodación de los funcionarios en otras dependencias o en la eventualidad de no ser posible por diversos factores como la falta de plazas para la reubicación o como se manifiesta en la motivación del acto administrativo expedido por el concejo municipal la falta de musculo financiero para soportar la carga que implicaba la nómina y prestaciones del personal, así como de los demás gastos adminsitrativos de la dependencia, se debio considerar una suma de dinero a modo de compesasión por la afectación a la estabilidad laboral de los funcionarios que quedarían truncadas.

De otro lado es imprudente ignorar el riesgo que implica desestimar la importancia y existencia de un órgano especilizado en el control fiscal de los gastos del ente territorial. Así las cosas con la actuación del Gobierno municipal no solo se estarían vulnerando las situaciones jurídicas expuestas en el párrafo anterior, sino que el gasto público quedaría sin control por lo que se estaría en manfiesta vulneración del interés general (representado en un gasto público eficiente que sastifaga plenamente las necesidades sociales y justificaciones para el gasto), pudiéndose generar a futuro otras demandas y situaciones negativas fruto de esta supresión. 
Ahora bien es claro que la identificación de estos riesgos, así como el deber de mitigar en el mayor grado posible el impacto que éstos generan son el resultado de una rigurosa preparación y planeación de la actividad administrativa a cargo de la Administración misma, siendo lo anterior la materilización del deber de prevenir el daño.

En el caso objeto de estudio resulta evidente que la administración en el ejercicio de una actividad claramente lícita incumplió su deber planear y por ende de prevenir los daños y afectaciones a las que efectivamente hubo lugar con ocasión del Acto Administrativo.

Por ello es procedente la reparación del daño causado por la Administración que aún teniendo el deber de prevenir no lo hizo, lo cual generó un evidente desequilibrio en las cargas públicas que deben soportar los administrados y los servidores públicos, expresado en la ruptura de las relaciones laborales a termino indefinido y con una duración determinanda como lo es el caso del accionante.

Incluso deberá a futuro responder por las demandas que se susiten por la falta de control al gasto público, ya que como se expueso en el apartado anterior los riesgos que se generan con ocasión de la ausencia del citado organo son previsibles y a su vez bajo la teoria de l presivión del daño, su ocurrencia debe ser indeminizada y soportada por la persona en mejor condición de soportarle.

Así las coas y bajo los arugmentos expuestos y en el marco fáctico del caso, asiste razón al petitum del demandante toda vez que si bien el Acto Administrativo que suprime la contraloría municipal se califica dentro del concepto en una actividad lícita de la Administración es evidente que existe un desequilibrio en las cargas públicas y el riesgo que esto representa era previsible para la amdinistración mediante el ejercicio de una adecuada planeación situación que finalmente no se vio consumada.

\section{CRÍTICA AL FALLO DEL CONSEJO DE ESTADO OBJETO DE ESTUDIO}

El Consejo de Estado en el fallo que nos ocupa en el presente analisis, decide como ya se presento en el trabajo negar las pretenciones del demandante, pero sin llegar verdaderamente ha pronunciarse de fondo sobre los problemas jurídicos propuestos. 
Lo anterior, resultado de una posición dominante durante muchos años en este cuerpo colegiado, en lo que refiere a la presición de la acción contenciosa seleccionada por la parte activa del litigio, pues al existir algun error sobre la selección de la tipología de la acción solo habrá lugar a un pronunciamento inhibitorio por parte de los magistrados y bajo dicho argumento una desestimación de las pretenciones.

Para el caso en concreto el Consejo de Estado decide confirmar la desción del tribunal de primera instancia, no bajo los mismos argumentos en los que el tribunal consideró que no existía mérito para conceder lo solicitado, sino por el contrario por presunamtnete no ser la acción contenciosa correcta para el caso. Afirmó el Consejo de Estado de manera facilista que el actor si bien no "pretendía" cuestionar la legalidad del acto sino las consecuencias que se desprende de éste, se evidencia de los diversos argumentos de la demanda que el actor cuestiona de fondo la legalidad del acto "En el sub examine, la Sala destaca que -contradictoriamente- el actor en su escrito de demanda controvierte los motivos aducidos en la exposición de motivos del citado acuerdo al afirmar:

Las razones que se adujeron en la exposición de motivos [del acuerdo 006 de 1996] presentada por el alcalde José Diego Villa Ramírez son de eminente carácter subjetivo, que sólo podrían entrarse a discutir si fuéramos a atacar el acto administrativo por causales como desviación del poder o la falsa motivación, o la aplicación indebida de la ley 136 de 1994. Que no es el presente caso (se destaca) (Consejo de Estado, Expediente No. 16421, 2007)

Bajo los anteriores entendidos procede entonces el Consejo de Estado a considerar improcedente la acción ya que efectivamente existe un cuestionamiento al Acto Administrativo, por lo que se debe hacer uso de la acción de Nulidad y Restablecimiento del Derecho (La cual pretende atacar la legalidad del acto y de prosperar expulsar al ordenamiento jurídico la actuación y sus efectos. De igual forma propende por la reparación de los derechos subjetivos afectados con ocasión del acto que se declara nulo y no como erróneamente se propuso en la demanda en uso de la Reparación Directa, así pues en palabras precisas de la Sala del Consejo de Estado la situación se:

Torna improcedente la acción, pues como lo ha dicho la jurisprudencia, si lo que se reprocha es la legalidad del acto 
el único medio idóneo para hacerlo es a través del contencioso de anulación. No es de recibo que el actor asegure que no cuestiona la legalidad en este proceso, pero implícitamente- deja entrever que bien podría hacerlo en otro como se desprende del texto de la demanda antes citado. (Consejo de Estado, Expediente No. 16421, 2007)

Resulta del anterior panorama, una crítica clara a las decisiones facilistas y sin mayor argumentación que aducir la improcedencia del medio (errores de forma) lo cual a juicio de la línea jurisprudencial de esta corporación hace inoportuno que el juez de lo contencioso administrativo decida de fondo, a pesar que como en el caso objeto tenga presente todos los elementos y presupuestos que dan a lugar a una condena patrimonial al Estado por responsabilidad extracontractual.

Entre el Consejo de Estado afirma "que en principio puede ventilarse esta controversia en sede de reparación directa, sin embargo, para que ello sea procedente es menester que se reúnan fundamentalmente las siguientes condiciones: i) Que se trate de un acto administrativo legal, esto es, que se trate de una actuación legítima de la administración; ii) Que se acredite que la carga impuesta al administrado sea anormal o desmesurada (rompimiento de la igualdad ante las cargas públicas o violación de la justicia distributiva)." Debe reflexionarse sobre esta posición, toda vez que el Acuerdo del Concejo de la Virginia no ha sido demandado y mucho menos anulado, por lo cual goza plenamente de su presunción de legalidad, la cual solo es afectable y desvirtuarle por parte del Juez de lo Contencioso Administrativo, quien deberá expresarlo asî de manera clara y motivada en una sentencia fruto de un medio de control de nulidad.

La situación expuesta no se cumple en el sentencia objeto de análisis ya que el Consejo de Estado, como ya se mencionó, se limita a exponer el argumento en el que el actor menciona la posibilidad de una ilegalidad del acto por la valoraciones subjetivas contenidas en éste, sin que esta "acusación" fuera una de las pretensiones de la demanda, que al fin y al cabo en Colombia es sobre lo que primordialmente deben pronunciarse los jueces junto con las excepciones de fondo propuestas por el demandado.

En conclusión, la decisión del Consejo de Estado refleja dos situaciones plausibles, la primera de ellas en lo que refiere al facilismo de la corporación judicial para resolver la situación pues procedió a confirmar 
el fallo de primera instancia pero bajo los argumentos de la improcedencia de la acción de reparación directa, ya que en los argumentos de la demanda a juicio del Consejo se esta cuestionando la legalidad del acto, lo que se consuma en una serie de sentencias inhibitorias sin un verdadero pronunciamiento de fondo. En un segundo lugar se hace la amplia discrecionalidad de la corporación a la hora de revisar y decidir sobre los casos sometidos a su control y revisión.

\section{CONCLUSIÓN}

El Estado Colombiano a partir de la constitución política de 1991 reconoce por voluntad del constituyente primario el deber de reparar los daños antijurídicos causados sobre los particulares con ocasión del ejercicio de sus funciones o la omisión de las mismas.

Es así que en el marco de la responsabilidad del Estado por los daños causados con ocasión del ejercicio de sus funciones se configura la existencia de la responsabilidad imputable por daño especial, es decir aquella responsabilidad que se predica, cuando el Estado con el ejercicio de una actividad legítima, afecta el equilibrio de las cargas públicas a las que están sometidos los particulares y afectando un bien jurídico tutelado.

El anterior planteamiento genera la existencia de una convergencia de principios y derechos que en el marco del desarrollo de una actividad legítima de la administración puedan suscitarse y desencadenar el desequilibrio de las cargas públicas a las que justamente deben someterse todos los administrados.

Se acude entonces, para evidenciar si efectivamente se han desequilibrado las cargas públicas, al método de ponderación de derechos y principios como respuesta al planteamiento del problema de investigación y la hipótesis formulada, evidenciándose que la ponderación es un ejercicio para advertir que derecho principio tiene cabida preponderante sobre el otro, dando como resultado en los dos ejercicios desarrollados respecto de la ponderación de derechos y principios en conflicto en el marco de la sentencia objeto análisis: Interés General, Seguridad Jurídica y Confianza Legítima. En cada uno de los ejercicios se obtienen resultados equidistantes, pero en todo caso completamente válidos según la carga y enfoque argumental que se imprima en cada ejercicio de ponderación, por lo que se puede concluir que el método de ponderación no es un medio que ofrezca una única 
respuesta y seguridad jurídica respecto de la solución en el marco del enfrentamiento de principios y derechos del caso objeto de análisis.

Como respuesta a la anterior situación se acude a otro medio para la revisión y solución del caso en análisis, siendo este la teoría de la prevención del daño, como deber de reparar por imputable a la parte que esté en mejor situación jurídica y práctica de cumplir mitigar y prevenir el daño, siendo éstos una expresión del deber de planear e identificar todas las aristas y aleas que en torno al ejercicio legítimo de la función pública se puedan suscitar.

Así pues queda claro que en el marco de estos procesos de reparación y responsabilidad por los daños antijurídicos causados en el marco del desarrollo de sus funciones, la responsabilidad y la condena de reparación deberá expresarse a través de la identificación de que efectivamente el Estado haya omitido la mitigación de los daños que con su actuar pudiere ocasionar, y no en la expresión de sentencias inhibitorias por interpretaciones desestimadas del procedimiento, el cual finalmente se encuentra sometido a las reglas sustanciales del derecho, reflexión que se obtiene de la crítica efectuada a la sentencia del Consejo de Estado analizada en el marco del presente trabajo.

\section{REFERENCIAS BIBLIOGRÁFICAS}

Clérico, L. (2010) Hacia un modelo de la ponderación orientado por reglas para la solución de conflictos de derechos fundamentales, en Escobar, C. Teoría y Práctica de la justicia constitucional. Ministerio de Justicia y Derechos Humanos. Quito

Consejo de Estado. 7 de abril de 1938, C.P. Carlos Lozano y Lozano. Bogotá.

Consejo de Estado, 21 de marzo de 1996, Rad. 3575, Bogotá

Consejo de Estado. 13 de diciembre de 2005. Expediente 24.671, M.P. Alier Hernández Enríquez. Bogotá

Consejo de Estado. 8 de marzo de 2.007, Expediente No. 16421, Consejera Ponente Dra. Ruth Stella Correa, Bogotá.

Colombia. Corte Constitucional. 11 de noviembre de 2.010. Sentencia de Revisión de Tutela T895. M.P. Dr. Nilson Pinilla Pinilla. Bogotá. 
Costa Rica. Corte Suprema de Justicia. Sala Constitucional. (5 de Agosto de 2.011) Sentencia No. 10375-11. San José, Recuperado de http://sitios.poder-judicial.go.cr/salaconstitucional/Constitucion\% 20Politica/Sentencias/2011/11-010375.html

Ruiz, W. (2013) Responsabilidad del Estado y sus regímenes. Bogotá: ECOE Ediciones

Vergara, L. (2013) La prevención en el derecho de la responsabilidad civil. Recuperado en: Cita online AR/DOC/11701/2003 